\title{
ESTUDIO DE LOS ESTILOS DE CRIANZA EN NIÑOS, NIÑAS Y ADOLESCENTES EN COLOMBIA: UN ESTADO DEL ARTE
}

\author{
Sandra Castillo ${ }^{1}$, Darwis Sibaja ${ }^{1}$, Lina Carpintero' ${ }^{1}$ Kelly Romero-Acosta² \\ Recibido: 11-05-2015 - Aceptado: 07-09-2015
}

\begin{abstract}
Resumen
Hablar de estilos de crianza es hacer mención a la manera como el padre educa a su hijo y la relación que hay entre ellos. Este artículo tiene como objetivo determinar el estado del arte de los estudios que consideran como una de sus variables los estilos de crianza en Colombia. Se realizó una búsqueda exhaustiva de artículos científicos en bases de datos y en revistas científicas teniendo en cuenta los siguientes criterios de inclusión: que se hubiesen realizado en Colombia y que entre sus variables de estudio estuviesen incluidos los estilos de crianza. Se estudiaron los resultados encontrados y a partir de estos se plantearán futuras investigaciones, centrados en los de estilos de crianza en Colombia.
\end{abstract}

Palabras clave: Estilos de crianza, niño, niñas, adolescente, Colombia.

\section{STUDY OF PARENTING STYLES ON CHILDREN AND ADOLESCENTS IN COLOMBIA: A STATE OF THE ART}

\begin{abstract}
Parenting styles is related to how parents educate his son or daughter and the relationship between them. This article aims to determine the state of the art of the study about parenting styles on children and adolescents y Colombia. An exhaustive search of scientific papers was conducted in databases and, also, in scientific journals considering the following inclusion criteria: to be held in Colombia and that among study variables they were included parenting styles. The findings from these and future research were raised taking into account the issue of parenting styles in Colombia.
\end{abstract}

Keywords: Rearing styles; children; adolescents; Colombia.

\footnotetext{
${ }^{1}$ Estudiantes X semestre del Programa de Psicología de la Corporación Universitaria del Caribe-CECAR. Sincelejo, Sucre, Colombia. ${ }^{2}$ Doctora en Psicopatología, psicóloga, docente-investigadora en Corporación Universitaria del Caribe -CECAR. Sincelejo, Sucre, Colombia; kelly.romero@cecar.edu.co
} 


\section{Introducción}

La interacción, la relación y la comunicación afectiva, hacen del sistema de la familia un complemento para la socialización de la crianza. También, las bases éticas y morales de dicho sistema determinan algunos estilos de crianza, y estos, a su vez, condicionan entre otras cosas, el desarrollo emocional y afectivo de los individuos, por tanto, los estilos de crianza suponen la base para formar individuos más o menos adaptativos a las demandas sociales, así se ha estudiado el presente tema en diversos países alrededor del mundo.

Cuando se habla de estilos de crianza se hace referencia a un conjunto de conductas ejercidas por los padres hacia los hijos (niño, niña), teniendo en cuenta que los padres son los principales responsables del cuidado y protección de los niños/niñas, desde la infancia hasta la adolescencia (Céspedes, 2008; Papalia, 2005; Sordo, 2009).

La familia puede considerarse como la primera escuela en la que el ser humano aprende acerca de sus valores o lo que es aceptado por las reglas de la sociedad en la cual vive. Según Goleman (1997), "la vida en familia es la primera escuela de aprendizaje emocional; es el crisol doméstico en el que se aprende a sentirse uno mismo y en donde se asimila la forma en que los demás reaccionan ante nuestros sentimientos". Por lo tanto, en la familia se aprende a entender los sentimientos de los otros y a responder hacia éstos.

Algunas entidades han intentado suplir las necesidades básicas de la familia a través de los orfanatos, asilos y variantes de estos, pero el ambiente familiar es el único capaz de brindar el amor y el afecto necesario para el buen desarrollo del ser, aunque muchas de las familias sean tan conflictivas que en vez de optimizar el crecimiento psicológico, físico, moral y social de una persona terminan haciendo lo contrario y convirtiéndolas en seres que son peligro para el resto de la comunidad (Goleman, 1997). En este sentido, es de vital importancia el estudio de los estilos de crianza en una comunidad, con el fin de conocer los factores más característicos que promueven ciertos estilos de crianza que no generan actitudes positivas y adaptativas en los individuos. Esto contribuiría a brindar estrategias a la comunidad para reflexionar sobre el estilo de la crianza que más conviene a los hijos, para una mejor adaptación social.

Cuando se conforma un sistema, en este caso la familia, se debe conocer la función de cada estructura y la manera cómo estas se relacionan entre sí y a la vez mirar cómo influye el ambiente social en ese sistema, es decir, cómo se ve inmerso el sistema (familia) en el supra sistema (ambiente).

La familia es el primer nivel de socialización de los seres humanos por en cuyo seno también se desarrolla la personalidad y los estados emocionales, en consecuencia se convierten en uno de los contextos más importantes para el desarrollo evolutivo de cada individuo. Según Bornstein (2002) y Torío (2003), nadie parece dudar de que la familia sea el contexto de crianza más importante en los primeros pasos de vida de la evolución de un individuo y es donde se adquieren habilidades sociales y comunicativas y los primeros hábitos que permitirán tomar la autonomía y las conductas decisivas para la vida.

Musitu, Román y Gracia (1988), consideran que los elementos que determinan los estilos de crianza contribuyen a una mejor práctica educativa, estas serían la estructura, el afecto, el control conductual, la comunicación, la transmisión de valores y los sistemas externos. Los cuatro primeros hacen alusión a las relaciones intrafamiliares o micro sistémicas, y las últimas hacen referencia a la dimensión social, siendo esta la que se extiende como análisis en el estudio de la socialización. La posición dentro de un supra sistema (ambiente social) explicará en gran medida la toma de postura y modos de actuación en un sistema (familia).

En investigaciones anteriores se ha encontrado que los estilos de crianza influyen en el desarrollo de sintomatología internalizante (ansiedad/depresión) (Steinberg, Blatt-Eisengart 
BÚSQUEDA - Julio / Diciembre de 2015 - No. 15 (64 - 71)

y Cauffman, 2006). Franco, Pérez y Pérez (2014) hallaron que algunas actitudes y pautas de crianza (tales como, grado de satisfacción, niveles de apoyo, compromiso con la crianza, entre otras) influyen significativamente en el desarrollo y mantenimiento de alteraciones emocionales en los hijos.

Según Rodrigo y Palacios (1998), los estilos o prácticas de crianza que pretenden los padres en cada familia sirven para modular y canalizar las conductas de los hijos en la dirección que ellos consideren adecuada. Estos estilos de crianza se basan en lo aprendido en la propia familia paterna y materna, por tanto, se transmiten de generación en generación a partir de la experiencia de vida familiar.

Patterson (2002), señala que las pautas de crianza son el principal determinante del comportamiento antisocial en el niño. En este caso en particular, los estilos de crianza son un mecanismo básico denominado condicionamiento de escape, según el cual un niño aprende a responder de forma aversiva para dar fin a los comportamientos agresivos de sus padres o hermanos. Por lo tanto confirma una vez más la importancia de investigar acerca de los estilos de crianza y sobre las repercusiones que esto acarrea consigo.

Los padres autoritarios y permisivos no propician un despliegue adecuado de habilidades sociales (Valiente, Fabes, Eisenberg y Spinrad, 2004), mientras que un estilo de crianza basado en el afecto y en el control inductivo, favorece el desarrollo de conductas socialmente adecuadas (Confalonieri y Giuliani, 2005; Isaza, 2012).

Colombia es un país con una situación socio-política marcada por el conflicto interno que lleva consigo efectos colaterales en todos los ciudadanos colombianos, ya sean víctimas directas del conflicto o no. La estructura familiar puede verse afectada por causa de muerte, desapariciones, torturas, violaciones -entre otras situaciones- vividas por uno o varios miembros de la familia. En este sentido, el estilo de crianza, considerado herencia familiar y cultural podría verse matizado por la vivencia de estos hechos altamente estresantes, que podrían producir posibles sentimientos de miedo, rabia y/o frustración de padres a hijos y de hijos a padres. Por esta razón, es de vital importancia estudiar sobre los estilos de crianza en las familias colombianas. Las preguntas que se generan a partir de esta reflexión son las siguientes: ¿Se suele estudiar sobre los estilos de crianza en Colombia? ¿Cuál es el estado de conocimiento de los estilos de crianza en este pais?.

El estado del arte sirve para estar al tanto de lo que se conoce y de lo que se desconoce acerca de un tema de estudio. Además, ayuda a orientar y a focalizar nuevas perspectivas de investigación. Por todo lo anterior, el objetivo del presente trabajo es determinar el estado del arte de los estudios que consideran como una de sus variables, los estilos de crianza en Colombia.

\section{Metodología}

Se realizó una búsqueda exhaustiva de artículos científicos en bases de datos y en revistas científicas tales como, Scopus, Scielo y Psicodoc, teniendo en cuenta los siguientes criterios de inclusión, 1. Que hayan sido realizados en Colombia y 2. Que entre sus variables de estudio estuvieran incluidos los estilos de crianza; con estas palabras claves se encontraron 218 artículos en total y solo cumplieron los criterios de inclusión ocho.

Posteriormente se realizó una segunda búsqueda en revistas indexadas en Colombia, con base en el documento publicado por Ardila y Pérez-Acosta (2010). En total se encontraron 66 artículos, de los cuales solo 4 cumplieron con los criterios de inclusión.

Finalmente se escogieron nueve artículos puesto que tres artículos de las revistas se repetían en las bases de datos consultadas. 


\section{Resultados}

Los resultados encontrados en esta revisión teórica fueron nueve investigaciones, una de tipo cualitativo, cinco de tipo cuantitativo y tres de reflexión teórica. A continuación se especifican las investigaciones encontradas en este estudio. La gráfica 1 muestra los artículos según el tipo de estudio utilizado y la tabla 1 aporta un resumen de los trabajos cualitativos y cuantitativos hallados en esta investigación.

1. "Un análisis intercultural de la socialización familiar y los valores en adolecentes" (Agudelo et al., 2001). Este estudio realiza una investigación con adolescentes, en el cual utiliza como variables: los estilos de socialización familiar, el facilismo y los valores. Es de tipo cuantitativo. La finalidad de esta investigación fue analizar de forma conjunta las posibles diferencias entre las variables ya mencionadas y los valores en las comunidades; en ella se utilizó la técnica de análisis discriminante. Dicha técnica permite conocer cuál es la contribución de cada una de estas variables y la diferenciación entre los distintos grupos.

2. "Crianza y comportamiento moral: un modelo mediacional' (Velásquez, et al., 2006). Es una investigación de tipo cuantitativo que se realizó con una muestra de 114 niños. El objetivo principal del estudio fue evaluar si el comportamiento de los padres se relacionaba con la conducta moral de sus hijos preadolescentes y si algunas atribuciones psicológicas mediaban en dicha relación. Los resultados de los análisis mostraron que el control restrictivo y los modelos de conducta moral de dichos padres sí se relacionaban con la conducta moral de sus hijos.

3. "La aceptación y el rechazo social en grupos de niños escolarizados entre los 9 y 11 años de edad" (Jaramillo, et al., 2006). En esta investigación de tipo cuantitativo se pretende identificar algunas variables individuales, familiares y escolares asociadas a la aceptación y al rechazo en grupos escolarizados, con edades comprendidas entre 9 y 11 años. Los estilos de crianza estudiados fueron: autoritario, permisivo, descuidado, sobreprotector y democrático. Para la recolección de datos se diseñó un cuestionario y una entrevista semiestructurada. Esta investigación se basa en 5 hipótesis, las cuales son: H1 la diferencia significativa entre los niños aceptados y los niños rechazados socialmente por sus compañeros y la capacidades que tienen estos para ejercer el control afectivo; H2: cuales son los déficit que presentan los niños rechazados o las malas conductas que contribuyen negativamente; H3: discriminar los estilos de crianza utilizados por las familias con mayor frecuencia que sufren rechazo en contraposición a los estilos de crianza utilizados por las familias en las cuales los niños no son rechazado;. $\mathrm{H} 4$ : mirar en las aulas el desarrollo de condiciones o rechazo social entre los alumnos y cuáles son los estilos utilizados por las familias a que pertenecen; y H5: mirar cuáles son las reacciones que los niños exteriorizan hacia sus compañeros.

Gráfico 1. Artículos según el tipo de estudio

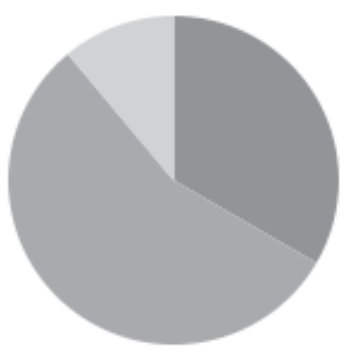

Reflexión teórica

Estudio de campo
cualitativo
Estudio de campo
cuantitativo

Fuente: Elaboración propia 
BÚSQUEDA - Julio / Diciembre de 2015 - No. 15 (64 - 71)

4. "Las representaciones sociales de género y castigo y sus índices en la corrección de los hijos" (Buitrago-Peña, et al., 2009). Es una investigación de diseño cualitativo que pretende aportar compresión sobre las representaciones sociales en relación con los correctivos implementados con los niños y niñas. Para este trabajo se utilizaron estudios de casos y entrevistas estructuradas. Las familias investigadas fueron de tipo nuclear, monoparental femenina o masculina. Se llegó a la conclusión de que el proceso de construcción de las representaciones sociales está dado por las formas de crianza y que las creencias, las pautas, las prácticas y los estilos de crianza influyen significativamente en la diferenciación de correctivos empleados con los hijos.

5. "El desempeño en habilidades sociales en niños, de dos y tres años de edad, y su relación con los estilos de interacción parental" (Isaza, et al., 2010) este es un estudio de tipo cuantitativo, en el cual se empleó la técnica de desarrollo de habilidades sociales en una muestra de infantes de dos a tres años de edad. Se consideró el desarrollo social como un proceso educativo gradual en el que los padres son la base para el potencial de dicho desarrollo social de sus hijos. Esta investigación fue de tipo descriptivo correlacional y se evaluaron diferentes estilos de crianza; tales como el autoritario, el equilibrado y el permisivo. Se concluyó que el estilo de crianza autoritario y el permisivo se asocian a bajos niveles de habilidades sociales, mientras que el estilo equilibrado propicia la representación de repertorio conversacional, de interés, solución de problemas y asertividad.

6. "La influencia del clima socio familiar y estilos de interacción parental sobre el desarrollo de habilidades sociales en niños y niñas" (Isaza, et al., 2012). Este estudio es de tipo cuantitativo y se propuso analizar la influencia de los estilos de interacción parental y del clima socio-familiar sobre el desarrollo de las habilidades sociales con una muestra de 108 niños y niñas. Se concluyó que a mayor presencia de acciones equilibradas y mayor clima de participación, los niños y las niñas presentan un mayor desempeño social y que, a mayores estrategias autoritarias, menor desarrollo de repertorios sociales.

7. "Pautas, prácticas y creencias acerca de crianza... ayer y hoy" (Izzedin y Pachajoa., 2009). Este trabajo analiza la relación entre las múltiples concepciones de la infancia y las pautas de crianza a través de diversos periodos de la historia. También enfatiza en los primeros años de vida y en la socialización familiar. Finalmente, plantea una reflexión acerca de las necesidades de la familia desde la psicología.

Tabla 1. Resumen de artículos de tipo investigación cualitativa y cuantitativa

\begin{tabular}{llll}
\hline \multicolumn{1}{c}{ Autores } & \multicolumn{1}{c}{ Muestra } & \multicolumn{1}{c}{ Variables estudiadas } & Tipo de estudio \\
\hline Buitrago-Peña, et al., 2009 & Adultos & Representaciones sociales de castigo y género. & Cualitativo \\
Jaramillo, et al., 2006 & Niños & Individuales, escolares y familiares. & Cuantitativo \\
Velásquez, et al., 2006 & Niños & $\begin{array}{l}\text { Crianza de los padres, modelo moral, empatía, } \\
\text { simpatía, autovaloración, comportamiento moral. }\end{array}$ & Cuantitativo \\
Isaza, et al., 2010 & Niños & $\begin{array}{l}\text { Estilos de interacción familiar y el desarrollo de } \\
\text { habilidades sociales. }\end{array}$ & Cuantitativo \\
Isaza, et al.,2012 & Niños & $\begin{array}{l}\text { Influencia de los estilos de interacción parental } \\
\text { y del clima social familiar sobre el desarrollo de }\end{array}$ & Cuantitativo \\
habilidades sociales. & Estilos de socialización familiar, familismo y \\
valores desde una perspectiva intercultural. & Cuantitativo \\
\hline
\end{tabular}

Fuente: Elaboración propia a partir de los datos del programa AAL. 
Sandra Castillo, et al. - Estudio de los estilos de crianza en niños, niñas y adolescentes en colombia

8. "Las pautas de crianza y desarrollo socioafectivo en la infancia" (Cuervo, 2010). Este trabajo se interesó por hacer una reflexión sobre la relación que existe entre pautas de crianza y el desarrollo socio-afectivo durante la infancia. También, considera la importancia de la familia para facilitar el desarrollo de conductas prosociales para la prevención de salud mental en la infancia como la depresión, la agresividad, la baja autoestima y la ansiedad.

\section{9. "Una nueva mirada hacia la construcción} de la paternidad" (Nieri, 2012). Este trabajo se interesa por estudiar y reflexionar sobre la paternidad, y tiene como propósito demostrar qué le ocurre al padre en el momento de la gestación, parto y puerperio de la mujer, concluye que las funciones de los padres van más allá de una tarea social y un sostén económico, y que es un acompañamiento emocional-afectivo en el proceso de gestión y de crianza del niño durante el desarrollo de su hijo, para dar identidad, amor, cuidado y seguridad dentro de la familia.

\section{Discusión}

El objetivo de este trabajo es determinar el estado del arte de los estudios que consideran como una de sus variables los estilos de crianza en Colombia. Los antecedentes que se han encontrado con respecto al tema son en su gran mayoría internacionales y los que se han realizado en Colombia se han llevado a cabo en el interior del país (Bogotá y Medellín), no hay estudios en la costa, mucho menos en el departamento de Sucre. También es importante señalar que las investigaciones en su gran mayoría toman como muestra principalmente a los niños más que a los padres. En este sentido sería interesante realizar estudios que tengan en cuenta el discurso de los padres o sus respuestas ante instrumento de cribado o screening.

Por otro lado, la mayoría de estudios relacionan los estilos de crianza con algunas variables relevantes en el proceso de crecimiento de los niños, tales como conducta pro social y/o habilidades sociales (Jaramillo, et al., 2006.,
Velásquez, et al., 2006., Isaza, et al., 2010 Isaza, et al., 2012., Buitrago-Peña, et al., 2009; Agudelo et al., 2001., Cuervo, 2010; Nieri, 2012; Izzedin et al., 2009), casi ninguno tiene en cuenta analizar la relación que existe entre los estilos de crianza y la presencia de problemas psicológicos, por ejemplo.

Jaramillo y sus colegas (2006), afirman que existen cinco clases de estilos de crianza que son: el autoritario, el permisivo, el descuidado, el sobre protector y el democrático. Esto coincide con otras investigaciones que también confirman que los padres manejan ciertos criterios y estilos al momento de educar o guiar a sus niños, y en su mayoría estás relacionados con la educación que han recibido los padres y que transmiten de forma evidente a sus hijos.

Para algunos países centro-americanos, los castigos se suministran con más frecuencia a los niños, mientras que en Colombia las dimensiones de castigo difieren según el sexo, la edad y el estrato socio económico. Por ejemplo, en Colombia, los castigos se le proporcionan con más reiteración a niños varones cuyas edades oscilan entre 0 y 14 años (Buitrago-Peña, CabreraCifuentes, Guevara-Jiménez, 2009). Con relación a este tema, podría estudiarse el tipo de castigo recibido por el menor y el estilo de crianza que más se relaciona con este modelo de prácticas. En muchas ocasiones el tipo de castigo puede incurrir en medio de una violencia intrafamiliar grave, lo que trae consigo castigos severos que rocen el maltrato infantil. Según un estudio realizado por el Instituto Nacional de Medicina Legal y Ciencias Forenses (2013) se realizaron 68230 peritaciones por violencia intrafamiliar en Colombia. Del total de los casos, el $65.58 \%$ correspondió a violencia de pareja, 14.23\% a violencia contra niños, niñas y adolescentes, $18.20 \%$ a violencia entre otros familiares y $2 \%$ a violencia contra el adulto mayor. Sería interesante profundizar más sobre el estilo de crianza más característico en familias que infligen castigos severos y que conviven en un ambiente con altos niveles de violencia intrafamiliar. ¿Cómo se educa a estos hijos? ¿Por qué se castiga de esa manera? ¿Cómo se relacionan los cuidadores con estos hijos e hijas?. 
BÚSQUEDA - Julio / Diciembre de 2015 - No. 15 (64 - 71)

La perspectiva socio culturalista enfatiza en el hecho de que los niños y las niñas necesitan de forma básica e importante el apoyo directo de sus padres para el adecuado desarrollo de su aprendizaje cognitivo y social, en relación con la interacción con el medio, También se encontró que la cultura influye de manera directa en el desarrollo de habilidades en el menor (Agudelo, Cava y Musitu, 2001).

Por otro lado, las pautas de crianza y el desarrollo afectivo y social de los niños están íntimamente relacionados con el estado mental de los padres, así lo confirman los antecedentes encontrados en el desarrollo de la presente investigación (Jaramillo et al., 2006; Velásquez et al., 2006; Isaza et al., 2010; Isaza et al., 2012; Buitrago-Peña et al., 2009; Agudelo et al., 2001, Cuervo, 2010; Nieri, 2012; Izzedin et al., 2009). Este tema es de vital importancia para el contexto colombiano, puesto que debido al conflicto interno y a la vivencia de situaciones con altos niveles de estrés, los trastornos mentales, en especial los trastornos de ansiedad y de depresión -es decir, internalizantes- tienen una alta prevalencia en el país. Esto según el estudio de salud mental que se realizó en el 2003 en el país, en el cual se encontró una prevalencia de $40,1 \%$ de trastornos mentales (Ministerio de Protección Social, 2005). En este estudio, los trastornos de ansiedad y los trastornos del estado de ánimo fueron los más prevalentes, esto es, un $19,3 \%$ y un $15 \%$, respectivamente.

A pesar de todo esto, en Colombia no se han hecho suficientes estudios acerca de este tema, es decir, focalizadas hacia la relación entre estilos de crianza y la prevalencia de problemas psicológicos, tales como ansiedad y depresión. Tampoco se han realizado suficientes investigaciones acerca de los estilos de crianza en general, especialmente en la costa, y mucho menos en el departamento de Sucre. Se recomienda llevar a cabo más investigaciones sobre los estilos de crianza en diferentes regiones colombianas y que se estudie la relación que existe entre el estilo de crianza y la aparición de problemas psicológicos.

\section{Referencias bibliográficas}

Agudelo, A., Cava, M. J., y Musitu, G. (2001). Un análisis intercultural de la socialización familiar y los valores en adolescentes. Escritos de psicología. 5, 70 - 80.

Bornstein, M. H. (2002). Parenting infants. En M. $\mathrm{H}$. Bornstein (Ed.), Handbook of Parenting 1. Children and Parenting, 12(2), 3-43.

Buitrago-Peña, M., Cabrera-Cifuentes, K. A., y Guevara-Jiménez, M. (2009). Las representaciones sociales de género y castigo y su incidencia en la corrección de los hijos. Educ.educ, 12(3) 53 - 71.

Céspedes, A. (2008). Educar las emociones, educar para la vida. Santiago, Chile: ed. B S.A.

Confalonieri, E. y Giuliani, C. (2005). Parental child-rearing practices and psychological adjustment of pre-school and school children. Eta-Evolutiva, 82, 67-73.

Cuervo, A. M. (2010). Pautas de crianza y desarrollo socioafectivo en la infancia. Diversitas. Perspectivas en psicología. 6(1), $111-121$.

Franco, N., Pérez, M. A., y Pérez, M. J. (2014) Relación entre los estilos de crianza parental y el desarrollo de ansiedad y conductas disruptivas en niños de 3 a 6 años. Revista de Psicología Clínica con Niños y Adolescentes. 1(2); 149-156.

Goleman, D. (1997). La inteligencia emocional. Barcelona: Kairós.

Isaza, L. V., y Henao, G. L. (2010). El desempeño en habilidades sociales en niños, de dos y tres años de edad, y su interacción con los estilos de interacción parental. Electronic Journal of Research in Educational Psychology. 8(3), 1051 - 1076.

Isaza, L. V., y Henao, G. L. (2012). Influencia del clima sociofamiliar y estilos de interacción parental sobre el desarrollo de habilidades 
sociales en niños y niñas. Persona. 15, 253 $-271$.

Izzedin, R. B., y Pachajoa, A. L. (2009). Pautas, práctica y creencias acerca de crianza... Ayer y hoy. Liberabit. 15(2), $109-115$.

Jaramillo, J. M., Díaz, K. O., Niño, L. A., Tavera, A. L., y Velandia, A. O. (2006). Factores individuales, familiares y escolares asociados a la aceptación y el rechazo social en grupos de niños escolarizados entre los 9 y 11 años de edad. Diversitas. Perspectivas en psicología. 2(2), 205 215.

Ministerio de Protección Social. Fundación FES Social (2005). Estudio nacional de salud mental-Colombia 2003, ISBN 95833-73-70-2, recuperado de http:// onsm.ces.edu.co/uploads/files/1243030_ EstudioNacionalSM2003.pdf.

Musitu, G., Roman, J. y Gracia, E. (1988). Familia y educación, Prácticas educativas de los padres y socialización de los hijos. Barcelona: Labor.

Nieri, L. (2012). Nueva mirada hacia la construcción de la paternidad. Revista Psicología Cientifica.com, 14(10).

Papalia, D. Wendkoss, S. y Duskin, R. (2005). Psicología del desarrollo, de la infancia a la adolescencia. 9a ed. México: McGrawHill Interamericana, 515.

Patterson, G. R. (1982). Coercitive family process. Eugene, OR: Castalia.
Patterson, G. R. (2002). The early development of coercive family processes. En J. B. Reid, G. R. Patterson y J. Snyder (Eds.), Antisocial behavior in children and adolescents: $A$ developmental analysis and model for intervention, 45(10), 25-44.

Rodrigo, M. J. y J. Palacios (1998). Familia y desarrollo humano. Madrid: Alianza Editorial.

Sordo, P. (2009). No quiero crecer. Viva la diferencia. Para padres con hijos adolescentes. Santiago de Chile: Editorial Norma. 232.

Steinberg, L., Blatt-Eisengart, I. y Cauffman, E. (2006). Patterns of competence and adjustment among adolescents from authoritative, authoritarian, indulgent and neglectful homes: a replication in a sample of serious juvenile offenders. Journal of Research on Adolescence, 16(1), 47-58.

Torío, S. (2003). Estudio socioeducativo de hábitos y tendencias de comportamiento en familias con niños de educación infantil y primaria en Asturias. Oviedo: Servicio de publicaciones, 10(4), 51-78.

Valiente, C., Fabes, R. A., Eisenberg, N. y Spinrad, T. L. (2004). The relations of parental expressivity and support to children's coping with daily stress. Journal of family psychology, 18(1), 97-106.

Velásquez, A. M., Barrera, F., y Bukowski, W. (2006) crianza y comportamiento moral: Un modelo mediacional. Suma psicológica. 13(2) $141-158$. 